\title{
Emergency department utilization and success rates for intraosseous infusion in pediatric resuscitations
}

\author{
Cheri Nijssen-Jordan, MD
}

\begin{abstract}
Objective: To determine the frequency of use and the success rates of intraosseous (IO) vascular access in the emergency department.

Design: A retrospective chart review.

Setting: A tertiary pediatric emergency department (ED) in a large urban centre.

Methods: ED resuscitations (ICD-9 code 996) occurring between Oct. 1, 1989, and Sept. 30, 1995, were identified by searching the ED database, inpatient database, ICU admission log and provincial medical examiner's database. From these, all cases involving $I O$ access were selected and comprised the study sample. Demographics, diagnosis, number of IO attempts, success or failure of IO placement, relevant times and patient outcomes were recorded on standard data forms. Frequency of use, success rates and performance times were reported.

Results: IO access was successful in 36 of $42(86 \%)$ patients. In total, there were 68 attempts, or 1.6 attempts per child. All but one child were less than 3 years of age. The median time to successful IO placement was 8 minutes. Two complications, both fractures, occurred in one patient, a 10-day-old neonate.

Conclusions: IO success rates were high despite infrequent use.

RÉSUMÉ ANALYTIQUE

Objectif: Déterminer la fréquence d'utilisation et le taux de succès de l'accès vasculaire intraosseux au département d'urgence.

Conception : Revue rétrospective de dossiers.

Cadre : Département d'urgence pédiatrique de soins tertiaires dans un grand centre urbain.

Méthodes: Les réanimations à l'urgence effectuées entre le premier octobre 1989 et le 30 septembre 1995 furent identifiées grâce à la recherche de la base de données de l'urgence, de celles des patients hospitalisés, du registre des entrées aux soins intensifs et de la base de données des médecins légistes provinciaux. Â partir de ces informations, tous les cas où la voie intraosseuse avait été utilisée furent sélectionnés pour former l'échantillon de l'étude. Les données démographiques, le diagnostic, le nombre de tentatives d'accès à la voie intraosseuse, le succès ou l'échec de l'intervention, les délais pertinents ainsi que le devenir des patients furent notés sur des formulaires de données standards. La fréquence d'utilisation, les taux de succès et les délais furent indiqués.

Résultats : L'accès intraosseux fut réussi chez 36 des 42 patients (86\%). Au total, il y eut 68 tentatives, soit 1,6 tentatives par enfant. Tous les enfants étaient âgés de moins de 3 ans, sauf un. Le délai moyen pour un accès intraosseux réussi était de 8 minutes. II y eut deux complications, toutes deux des fractures, chez un nouveau-né âgé de 10 jours.

Conclusions : Les taux de succès de l'accès vasculaire intraosseux étaient élevés malgré un recours occasionnel à cette méthode.
\end{abstract}

Key words: intraosseous, pediatric, emergency department, resuscitation, infusion 


\section{Introduction}

Intraosseous (IO) infusions were first used in the 1920s when Drinker and colleagues ${ }^{1}$ demonstrated that fluids administered into the marrow cavity did reach intravascular circulation. More recently, IO lines have been used to deliver blood products to trauma and burn victims, to infuse drugs for rapid sequence intubation, and to achieve vascular access during fetal surgery and neonatal resuscitation. ${ }^{2-9}$ Despite its growing use in diverse situations, IO success rates are poorly documented in the hospital setting. This retrospective study was performed to assess the use of intraosseous vascular access at a pediatric tertiary care centre. The study objectives were to determine the frequency of IO use, its success rate, the time necessary to perform the procedure and the related outcomes.

\section{Methods}

\section{Design and setting}

This was a retrospective chart review performed in a tertiary pediatric emergency department (ED) in a large urban centre.

\section{Subjects}

Patients who underwent intraosseous line placement attempts during resuscitation in the Alberta Children's Hospital ED between Oct. 1, 1989, and Sept. 30, 1995, were eligible for inclusion. Because the study addresses ED practice, patients were excluded if their IO attempts were performed by prehospital care providers, physicians at other institutions or inpatient medical staff.

All patients who undergo resuscitation in the study hospital's ED are assigned the ICD-9 resuscitation code (996); therefore it was possible to identify eligible cases by searching the ED database. To ensure that no ED resuscitations were missed, the hospital's inpatient database, the intensive care unit admission log, and the Alberta Chief Medical Examiner's database (the latter to identify children who presented to the ED but died prior to hospital admission) were also searched. Standard nursing practice is to chart all resuscitation procedures; therefore it was possible to identify all patients who underwent intraosseous placement attempts.

\section{Data acquisition}

Charts that met study eligibility criteria were abstracted using standardized data forms. The following critical data were recorded: patient age, primary diagnosis, attending physician, time resuscitation was initiated, number of intravenous (IV) attempts, number of IO attempts, anatomic site of IO attempts, success or failure of IO placement, time of successful IO placement, return of spontaneous circulation, and survival to hospital discharge.

\section{Definitions}

Critical times were obtained from the resuscitation record. Number of IV and IO attempts were identified through a review of physician and nursing notes. Success in obtaining IO access was defined as the ability to aspirate bone marrow or to infuse saline without palpable extravasation. Return of spontaneous circulation (ROSC) was defined as a recordable heart rate with palpable pulses. Children who were discharged home with a care provider were deemed to have survived to hospital discharge (SURV). Missing data were reported as "unknown."

\section{Statistics}

Descriptive statistics, including means, medians, ranges and interquartile ranges were calculated for procedural success rates and temporal outcomes. $\mathrm{Chi}^{2}$ analysis was used to determine the statistical significance of observed differences in categorical outcome variables.

\section{Results}

\section{Subjects}

During the 6-year period, 57 patients underwent intraosseous access attempts. Fifteen were excluded because their IO attempts were performed outside the ED, leaving 42 in the study sample. Study patients ranged from 4 days to 10 years of age, with 41 of 42 (98\%) being less than 3 years old. They had a variety of primary diagnoses, including sudden infant death syndrome, congenital heart disease, supraventricular tachycardia with congestive heart failure, respiratory distress, bronchopulmonary dysplasia, trauma, hypovolemic shock, septic shock, and oncological complications. All attending physicians were Paediatric, Anaesthesia, or Emergency Medicine (Royal College or CCFP-EM) specialists.

\section{Interventions}

During the 6-year study period, 42 children had 68 intraosseous attempts (mean, 1.6 attempts per child; range 1-4). Figure 1 shows that this is an average of 7 children per year (range, 4-9), or 0.2 per 1000 ED visits. IO access was ultimately successful in 36 patients $(86 \%)$, and the success rate did not differ based on patient age (Fig. 2) or physician IO frequency of use (Table 1). Median time from initiation of resuscitation to successful IO placement was 8 minutes (IQR, 4-25 $\mathrm{min}$ ). Sixty attempts (88\%) involved the proximal tibia, 2 (3\%) involved the distal femur, and in 6 cases (9\%) no anatomic location was specified. All patients had 
undergone multiple intravenous attempts prior to IO placement. In 30 cases, IV access was achieved -8 times before the IO attempt and 22 after.

\section{Outcomes}

Nineteen patients (45\%) failed resuscitation, 10 (24\%) died in hospital after return of spontaneous circulation and 13 (31\%) survived to hospital discharge (Fig. 3). All 13 sur-

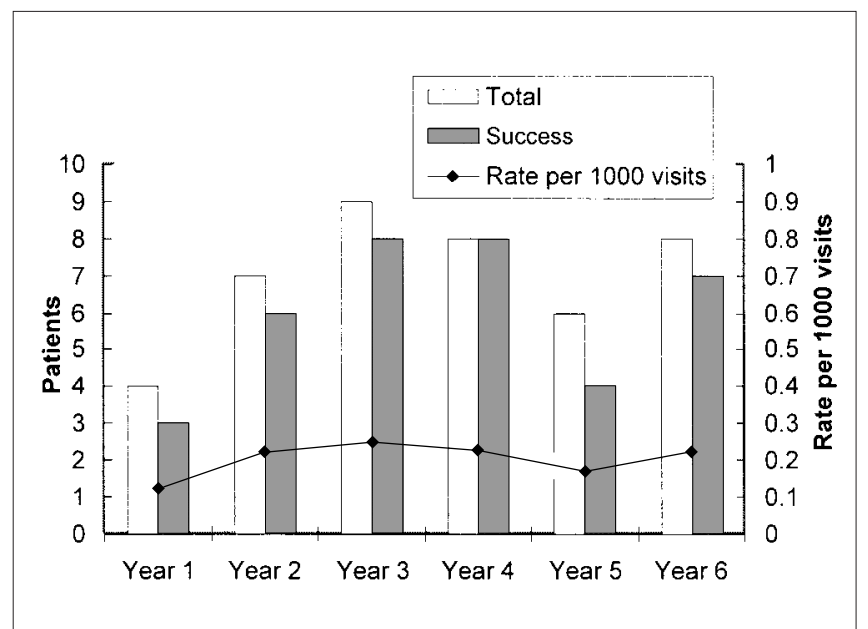

Fig. 1. Children undergoing intraosseous access, Oct. 1, 1989, to Sept. 30, 1995

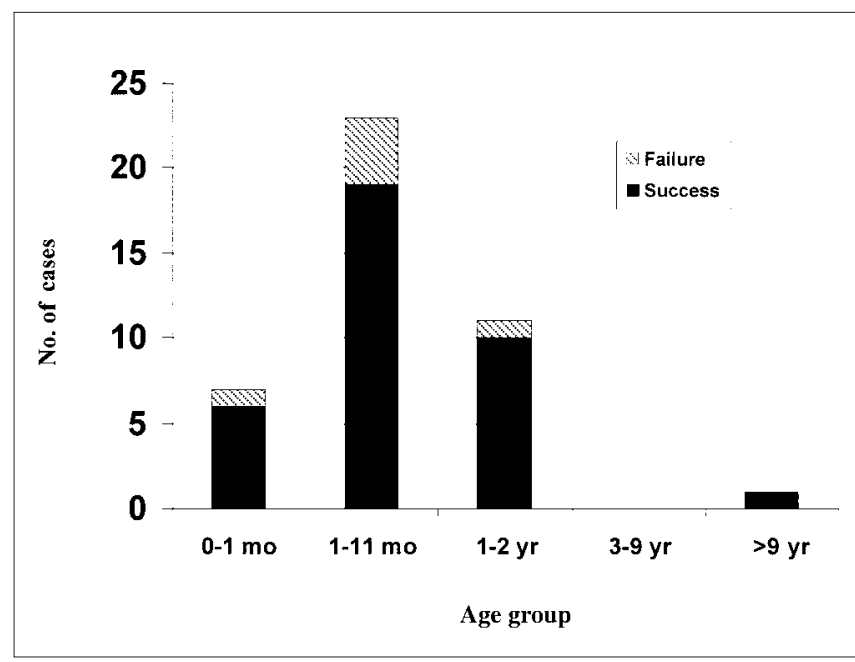

Fig. 2. Intraosseous success by age group

\begin{tabular}{|c|c|c|c|}
\hline & Group A* & Group B† & $p$ value \\
\hline Success/attempts & $15 / 24(63 \%)$ & $28 / 44(64 \%)$ & 0.93 \\
\hline Success/patients & $11 / 14(79 \%)$ & $25 / 28(90 \%)$ & 0.35 \\
\hline
\end{tabular}

vivors were in shock (hypovolemic, septic or cardiogenic) but not in full cardiac arrest at the time of IO insertion. Overall, 11 of $36(31 \%)$ patients with successful IO insertion survived versus 2 of $6(33 \%)$ with failed IO insertion ( $p$ $=0.89$ ). Complications were limited to 2 fractures that occurred in the same patient, a 10-day-old infant with coarctation of the aorta. The first fracture occurred in the proximal tibia, the second in the distal femur, and neither IO attempt was successful. No complications were documented in patients with successful IO placement.

\section{Discussion}

Intraosseous access is touted as a rapid, easy, safe, and potentially lifesaving procedure. ${ }^{10-12}$ However, given the infrequency of pediatric resuscitation, it is not surprising that experience with this technique is limited. In the study hospital, the number of resuscitations per year that required attempts at IO access (7) reflects the rarity of pediatric resuscitation as well as the small number of patients who do not already have vascular access.

In this setting, $64 \%$ of IO attempts were successful, patients underwent an average of 1.6 attempts, and $86 \%$ of patients had successful IO placement. Statistical differences in success rates between physicians who used the procedure more than twice (Table 1; Group B) and physicians who used it one to two times during that time period (Table 1; Group A) could not be demonstrated. These results are similar to other published data. In 1993, Glaeser and colleagues ${ }^{13}$ reported a 5-year prehospital experience with intraosseous infusions in children and adults. In that study, $70 \%$ of IO attempts were successful and $76 \%$ of patients underwent successful IO placement. The authors concluded that prehospital care providers can maintain good success rates despite infrequent use. They were also unable to demonstrate higher success rates for paramedics who used the procedure more frequently. In 1986, Kantor and coworkers ${ }^{14}$ proposed a resuscitation protocol suggesting that IV access should be achieved within 5 minutes. In their series of 36 resuscitations, 6 patients required IO attempts and 5 of these were successful. No other published studies report hospital or ED intraosseous utilization and success rates.

Interestingly, physicians in the present study delayed the initiation of IO access beyond the current Pediatric Advanced Life Support recommendations of 90 seconds or 3 intravenous attempts. This may indicate discomfort with the IO process, or it may reflect the confidence and persistence of tertiary care providers in attempting to achieve peripheral or central IV access. The median time of 8 min- 


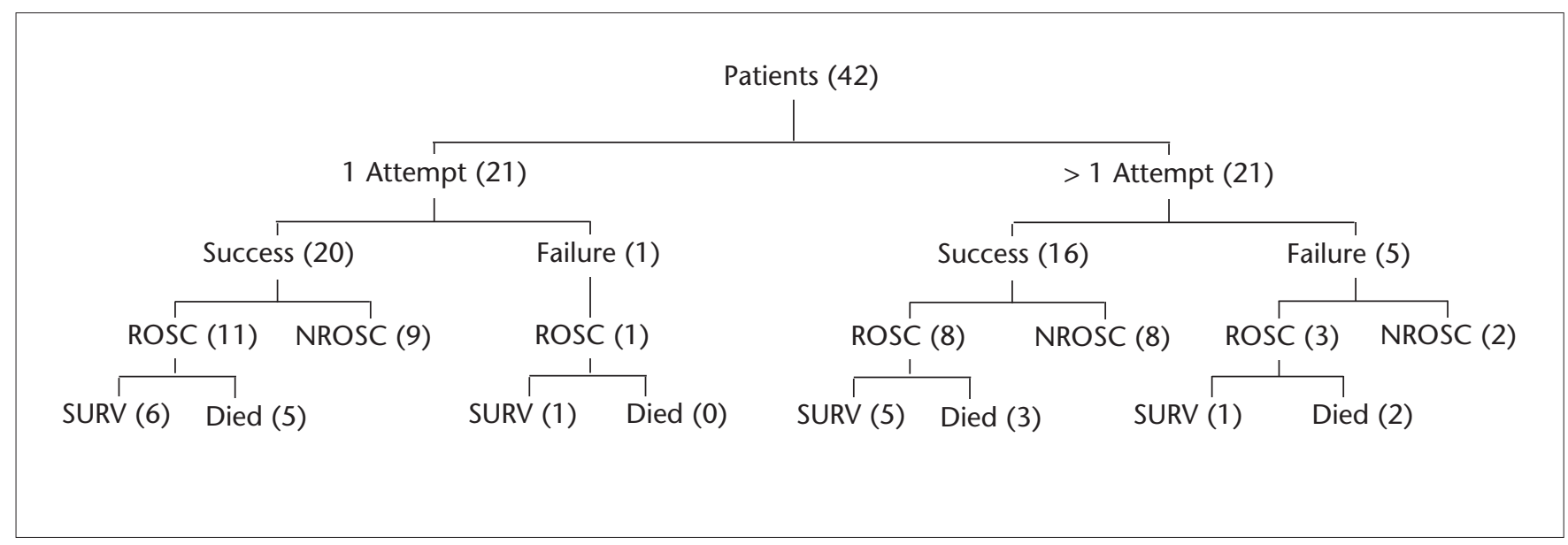

Fig. 3. Intraosseous use related to survival. SURV = survival to discharge; ROSC = return of spontaneous circulation but died before discharge; NROC = no return of circulation

utes to IO access overestimates therapeutic delays, since this interval includes time to decision and procedural time. In addition, some of the prolonged IO access times occurred because an initial IV line failed and secondary (late) access was required.

The only significant complications in this series were 2 fractures that occurred in a 10-day-old infant. The first of these, in the proximal tibia, was possibly due to faulty positioning and the second, in the distal femur, occurred during the rapid manual pressure infusion of a fluid bolus. It seems likely that the latter fracture was related to excessive pressure generation in the marrow space. Unfortunately, there is no human data on the ability of neonatal bone to withstand pressure peaks during manual IO fluid infusion. This suggests that fractures are an important outcome to monitor in infants who undergo IO access with or without pressure infusion.

A critical question that remains unanswered is: Does rapid IO access improve outcomes after pediatric arrest? In this series, outcomes were similar regardless of whether IO access was successful or not, but with only 6 cases in the "failure" group, the evidence is essentially anecdotal. Moreover, other potentially confounding factors, including differences in pre-hospital care, arrest duration prior to IO insertion, airway status, and care-provider experience prevent definitive conclusions. Given the secondary importance of parenteral medications (relative to airway management) and uncertainty about the efficacy of these medications in cardiac arrest, it is clear that a huge study would be required to demonstrate a survival benefit of IO infusion, and such a study is not likely to be attempted. Despite this uncertainty, there are probably subgroups (e.g., patients in impending cardiopulmonary arrest) who would benefit from rapid IO access by experienced care providers.

\section{Study limitations}

It is believed that the search strategy for this study was comprehensive and that all cases of IO insertion were identified; however, some cases may have been missed if they were undocumented in the medical record. Based on the retrospective nature of this study, time to IO access could be determined, but time to IO decision and time to perform the procedure could not. Because survivors were not followed after hospital discharge, it is possible that late complications in some patients were missed. Successful and failed insertions were identified, but the reasons for these cannot be speculated upon.

\section{Conclusions}

In this study, (per patient) intraosseous success rates were high despite a small number of attempts over a prolonged time period. These data suggest that successful IO access with a low complication rate can be accomplished despite infrequent use. Quality improvement mechanisms may be warranted to ensure the timely use of intraosseous infusions in patients at risk.

\section{References}

1. Drinker CK, Drinker KR, Lund CC. The circulation in the mammalian bone-marrow. Am J Physiol 1922;62:1-92.

2. Josefson A. A new method of treatment — intraossal injections. Acta Medica Scandinavica 1934;LXXXI:550-64.

3. Tocantins LM, O'Neill JF. Infusion of blood and other fluids into the circulation via the bone marrow. Proceedings from the Society of Experimental Biology and Medicine 1940;45:782-3.

4. Tocantins LM, O'Neill JF. Infusions of blood and other fluids into the general circulation via the bone marrow: techniques and results. Surg Gynecol Obst 1941;73:281-7. 
5. Arbeiter HI, Greengard J. Tibial bone marrow infusion in infancy. J Pediatr 1944;25:1-16.

6. Spivey WH, Lathers CM, Malone DR, Unger HD, Bhat S, McNamara RN, et al. Comparison of intraosseous, central and peripheral routes of sodium bicarbonate administration during CPR in pigs. Ann Emerg Med 1985;14:1135-40.

7. Medina FA. Rapid sequence induction/intubation using intraosseous infusion of vecuronium bromide in children. Am J Emerg Med 1992;10:359-60.

8. Jennings RW, Adzick NS, Longaker MT, Lorenz HP, Estes JM, Harrison MR. New techniques in fetal surgery. J Pediatr Surg 1992;27:1329-33.

9. Guy J, Haley K, Zuspan SJ. Use of intraosseous infusion in the pediatric trauma patient. J Pediatr Surgery 1993;28:158-61.

10. Chameides L, editor. Pediatric advanced life support. Wash- ington: American Heart Association/American Academy of Pediatrics; 1997.

11. Spivey WH. Intraosseous infusions. J Pediatr 1987;111:639-43.

12. Strange G, editor. Advanced pediatric life support. The pediatric emergency medicine course. Washington: American Heart Association/American Academy of Pediatrics; 1998.

13. Glaeser PW, Hellmich TR, Szewczuga D. Five-year experience in prehospital intraosseous infusions in children and adults. Ann Emerg Med 1993;22:1119-24.

14. Kanter RK, Zimmerman JL, Strauss RH, Stoeckel KA. Pediatric emergency intravenous access: evaluation of a protocol. AJDC 1986;140:132-4.

Correspondence to: cheri.nijssen-jordan@crha-health.ab.ca

\section{CJEM ${ }_{E^{13}} \mathrm{JCMU}$}

\section{The best place for your landmark article}

$\mathrm{N}$ ew journals face a critical catch-22. The National Library of Medicine (MEDLINE) will not index a new journal until it is publishing high quality scientific material, but authors would rather not submit high quality scientific articles to a new journal until it is indexed. Consequently, one of the big challenges for any new journal is to attract good authors and high quality articles.

Authors want their work to reach the largest possible audience, and want their article catalogued in a searchable electronic database so future readers and researchers can reference it easily. While CJEM is not yet an indexed journal, we have addressed both these issues. In terms of visibility, CJEM is distributed to all Canadian emergency physicians and residency programs, giving it the largest Canadian readership of any EM journal. In addition, CJEM is distributed to over 150 US residency programs and to Canadian expatriates and "prominent" emergency physicians in Europe, Asia, Africa, Australia and the Middle East. Current CJEM distribution is 7,500 copies per issue, which compares favourably to other EM journals. Only Annals of Emergency Medicine has a substantially larger readership base and, of course, it does not come with an attractive teal cover or any kind of decent humour section.

To ensure that our articles reach an international audience, CJEM is the only EM journal freely available in full text format on the World Wide Web. This increases North
American exposure and international penetration. While the exact level of the increase is unclear, some marketing experts suggest that a journal published on the Web will reach up to 10 times as many readers as the corresponding hard-copy journal. We therefore believe that articles published in CJEM will be read by more people than articles published in any other EM journal.

In addition, complimentary CJEM issues go to Drs. Jerry Hoffman and Rick Bukata, so that our articles can be reviewed on Emergency Medical Abstracts (EMA) tapes and enter the EMA database - perhaps the most useful article database for emergency physicians.

Soon, after our first full year of publication, we become eligible to apply for National Library of Medicine indexing. While this is not a "sure thing," we have been told that, based on the quality of the journal so far, CJEM is likely to be accepted. If so, then articles published prior to indexing will be retrospectively added to the MEDLINE database. In the interim, our articles are available to anyone who performs an Internet search.

For now, the New England Journal of Medicine remains the more prestigious of the 2 journals, but those days are numbered. If you have a landmark article and no connections in Boston, remember that CJEM offers peer-review publication, a large reading audience, and — maybe - a free tee-shirt (we're still thinking about this one). 